\title{
Factores abióticos que influencian la germinación de seis especies herbáceas de la zona árida de Chile
}

\author{
Abiotic factors effects influencing the germination of six herbaceous species \\ of Chilean arid zone
}

\author{
PAOLA A. JARA ${ }^{1,2,3^{*}}$, GINA ARANCIO ${ }^{1,2}$, RAÚL MORENO ${ }^{1}$ \& MARTÍN R. CARMONA ${ }^{2,3}$ \\ ${ }^{1}$ Departamento de Biología, Facultad de Ciencias, Universidad de La Serena, La Serena, Chile \\ ${ }^{2}$ Instituto de Ecología y Biodiversidad (IEB), Casilla 653, Santiago, Chile \\ ${ }^{3}$ Fundación "Senda Darwin”, Casilla 114-D, Santiago, Chile; \\ * e-mail para correspondencia: pjarancio@yahoo.com
}

\begin{abstract}
RESUMEN
La zona árida del norte de Chile presenta un clima seco que impide la emergencia masiva de especies vegetales, excepto cuando se producen escasas e irregulares precipitaciones que modifican las características de aridez y estimulan la germinación de semillas. Los principales factores externos que modifican estructuras y condiciones internas de las semillas son el tiempo de hidratación, iluminación, temperatura y escarificación. En este trabajo se abordaron dos interrogantes: (a) ¿la germinación de semillas de seis especies de zonas áridas es regulada por diferentes factores externos o presentan respuestas comunes a una combinación de estímulos? y (b) ¿existe correspondencia entre las condiciones germinativas establecidas en laboratorio y las determinadas in situ? Para resolver estas interrogantes se sometieron seis especies herbáceas endémicas y nativas del norte de Chile (Cistanthe salsoloides, Leucocoryne purpurea, Pasithea coerulea, Placea amoena, Schizanthus litoralis y Trichopetalum plumosum) a dos experimentos germinativos con combinaciones factoriales de tiempo de hidratación, temperatura e iluminación. Solo $S$. litoralis fue sometido a un experimento adicional de escarificación y deshidratación. Los resultados determinaron que todas las especies en estudio presentaron respuestas germinativas comunes frente a determinados factores externos, con una máxima germinación al sobrepasar un umbral de $96 \mathrm{~h}$ de hidratación y permanecer a niveles de temperatura de 10 a $25{ }^{\circ} \mathrm{C}$. La respuesta frente a iluminación fue especie dependiente. Schizanthus litoralis solo responde a estímulos externos previo a ser escarificada su semilla. Además, se determinó que existe concordancia entre los factores germinativos determinados en laboratorio y las condiciones naturales de germinación. Por lo tanto, las semillas de las especies en estudio presentan umbrales germinativos semejantes, y las técnicas de germinación en laboratorio deben ser determinadas por las características ambientales del lugar de procedencia y de la época de germinación natural.
\end{abstract}

Palabras clave: germinación, hidratación, temperatura, iluminación, zonas áridas.

\begin{abstract}
The arid zone of northern Chile has a dry climate that prevents the massive emergency of plant species. The exception to this general trend arises when scarce and irregular rainfall events occur, modify the environmental humidity, and stimulate the germination of seeds. The main external factors that modify the internal nature of the seeds are the hydration time, light, temperature and scarification. In this work two questions were addressed: (a) is the germination of seeds of arid zones regulated by independent external factors or by a combined array of stimuli? and (b) do exist correspondence between laboratory and in situ germinative conditions?. Seeds of six native and endemic herbaceous species of the north of Chile (Cistanthe salsoloides, Leucocoryne purpurea, Pasithea coerulea, Placea amoena, Schizanthus litoralis y Trichopetalum plumosum) were subjected to two germination experiments, with factorial combinations of hydration time, temperature, light, dehydration and scarification. Schizanthus litoralis, was subjected to an aditional scarification-dehydration experiment (experiment 3). Results showed a common response of all the species in study to certain external factors. Maximum germination percentages were reached when exceeding a threshold of $96 \mathrm{~h}$ of hydration and at temperatures of 10 to $25{ }^{\circ} \mathrm{C}$. Light response was species-dependent. Agreement was found between the germinative conditions determined in laboratory and natural conditions of germination. Therefore, the seeds of plant species of arid zones display similar germinative thresholds and the techniques of germination in laboratory must be determined by the environmental characteristics of the place of origin and the time of natural germination.
\end{abstract}

Key words: germination, hydration, temperature, light, arid zones. 


\section{INTRODUCCIÓN}

La condiciones áridas de la zona norte de Chile (Gengler-Nowak 2002), se ven modificadas periódicamente por el fenómeno El Niño/ Oscilación del Sur (ENOS), donde se produce un incremento en las precipitaciones (Romero 1985, Toledo \& Zapater 1989, Cepeda 1995). Este fenómeno estimula la emergencia de especies vegetales, adaptadas a sobrevivir en un constante estado de tolerancia y latencia (Toledo \& Zapater 1989), respuesta biológica conocida como "Desierto Florido". En esta zona árida las semillas al ser liberadas, pueden germinar inmediatamente o caer en un estado de latencia innata o impuesta (Vegis 1964, Baskin \& Baskin 1978, 1981, 1984, 1993, 1998, Karssen 1980, Froud-Williams et al. 1984). La latencia es un mecanismo que retrasa la germinación hasta que las condiciones internas (madurez embriológica, eliminación de inhibidores o permeabilidad de la testa) y/o externas (humedad, temperatura e iluminación) sean óptimas para que las semillas germinen y se establezcan las plántulas (Fenner 1985, Mayer \& Poljakoff 1989, Inouye 1991, Bailin \& Foley 1997).

Al analizar la emergencia masiva de especies vegetales herbáceas en zonas áridas, que se relaciona con factores externos ambientales específicos, surgen dos interrogantes: (a) ¿la germinación de semillas de seis especies de zonas áridas es regulada por diferentes factores externos o presentan respuestas comunes a una combinación de estímulos?, y (b) ¿existe correspondencia entre las condiciones germinativas establecidas en laboratorio y las determinadas in situ? Para responder estas interrogantes, se realizaron análisis germinativos con una combinación factorial de tiempo de hidratación, temperatura e iluminación en seis especies herbáceas pertenecientes a la zona árida del norte de Chile.

\section{MATERIALES Y MÉTODOS}

\section{Especies de estudio}

Para realizar los estudios germinativos, se utilizaron seis especies herbáceas: (Cistanthe salsoloides (Bar.) Car. ex Her., Leucocoryne purpurea Gay, Pasithea coerulea (Ruiz et Pav.) D. Don, Placea amoena Phil., Schizanthus litoralis Phil. y Trichopetalum plumosum (Ruiz et Pav.) JF Mac.), pertenecientes a la zona árida del Norte de Chile (Marticorena \& Quezada 1985, Squeo et al. 2001) (Fig. 1, Tabla 1).

TABLA 1

Características de las seis especies en estudio; $\mathrm{O}=$ origen $(\mathrm{E}$ : endémica, $\mathrm{N}$ : nativa); $\mathrm{EC}=$ estado de conservación (FP: fuera de peligro de extinción, VU: vulnerable); $\mathrm{D}=$ distribución; $\mathrm{A}=$ altitud

Characteristics of the six species studied; $\mathrm{O}=$ origin $(\mathrm{E}$ : endemic, $\mathrm{N}$ : native); $\mathrm{EC}=$ conservation status (FP: out of extinction danger, $\mathrm{VU}$ : vulnerable); $\mathrm{D}=$ distribution; $\mathrm{A}=$ altitude

\begin{tabular}{|c|c|c|c|c|c|}
\hline Especie & Familia & $\mathrm{O}$ & $\mathrm{EC}$ & $\mathrm{D}$ & A (m) \\
\hline L. purpurea Gay & Alliaceae & $\mathrm{E}$ & $\mathrm{VU}$ & IV & $50-2.700$ \\
\hline P. amoena Phil. & Amaryllidaceae & $\mathrm{E}$ & $\mathrm{VU}$ & IV a RM & 1.500 \\
\hline P. coerulea (Ruiz et Pav.) D. Don & Hemerocallidaceae & $\mathrm{N}$ & FP & II a X & $50-1.500$ \\
\hline T. plumosum (Ruiz et Pav.) JF Mac. & Lomandraceae & $\mathrm{E}$ & FP & IV a RM & $50-900$ \\
\hline C. salsoloides (Bar.) Car. ex Her. & Portulacaceae & $\mathrm{N}$ & FP & II a IV & $600-3.000$ \\
\hline S. litoralis Phil. & Solanaceae & $\mathrm{E}$ & FP & IV a V & $50-3.000$ \\
\hline
\end{tabular}

Números romanos en la columna D corresponden a regiones administrativas de Chile 

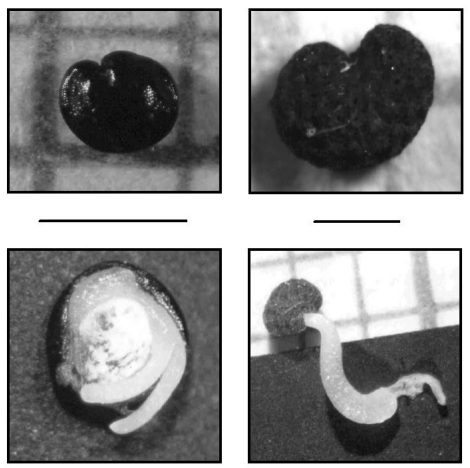

(A)

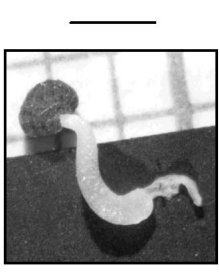

(B)
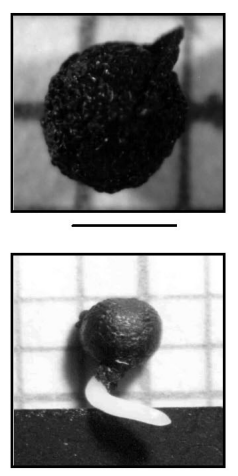

(C)
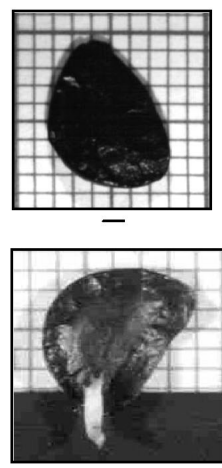

$-$

(D)
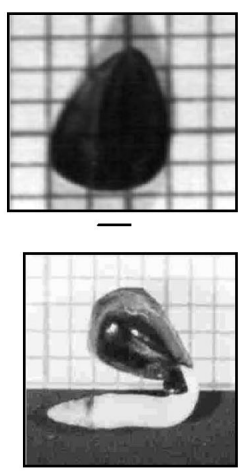

(E)
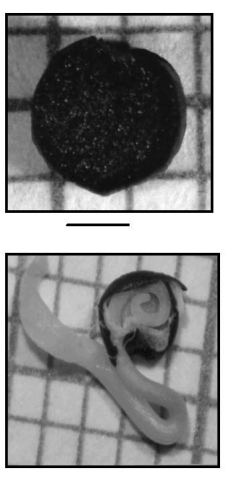

(F)

Fig. 1: Morfología externa de semillas no germinadas (superior) y germinadas (inferior) de las seis especies en estudio de la zona árida de Chile; (A) C. salsoloides, (B) S. litoralis, (C) L. purpurea, (D) P. amoena, (E) P. coerulea y (F) T. Plumosum $(1 \mathrm{~mm}=-$-).

External morphology of non germinated (upper) and germinated (lower) seeds of species under study of the arid zone of Chile; (A) C. salsoloides, (B) S. litoralis, (C) L. purpurea, (D) P. amoena, (E) P. coerulea and (F) T. plumosum $(1 \mathrm{~mm}=--$ ).

\section{Recolección de semillas}

Entre los meses de enero y febrero de 1997 se colectaron frutos maduros de diferentes individuos de cada especie en estudio. Las semillas acumuladas por especie fueron almacenadas en bolsas de papel en el interior de una cámara termorregulada a $25{ }^{\circ} \mathrm{C}$, durante 60 días hasta la realización de las pruebas germinativas.

\section{Análisis de viabilidad}

Se escogieron al azar 100 semillas por especie, las que fueron sometidas a una prueba de viabilidad pregerminativa con solución de Cloruro de Tetrazolio al 1 \% (Moore 1973), según protocolo de Booth \& Hendy (1993). Las semillas no germinadas después de los experimentos fueron sometidas a una prueba de viabilidad postgerminativa.

\section{Experimentos de germinación}

Se realizaron dos experimentos independientes y consecutivos que consideraron tratamientos germinativos con distintas combinaciones de tiempos de hidratación, temperatura e iluminación. Para cada tratamiento, se utilizaron 100 semillas por especie, divididas en cuatro réplicas con 25 semillas cada una. Los experimentos consistieron en dos etapas:
(1) hidratación, las semillas fueron sumergidas en agua destilada por diferentes períodos y (2) germinación, después de ser hidratadas las semillas fueron trasladadas a cápsulas petri con papel absorbente húmedo. En ambas etapas se mantuvieron los niveles de temperatura e iluminación preestablecidos para cada tratamiento. En cada experimento las etapas de hidratación y germinación, se realizaron en tres cámaras termorreguladas acondicionadas a las temperaturas asignadas. La luminosidad fue manipulada mediante oscurecimiento de las cápsulas petri con papel aluminio en el interior de cada cámara. Durante un período de ocho semanas se registró cada 48 horas el número de semillas germinadas. Se consideraron como semillas germinadas aquellas que presentaron la aparición de radícula y zona vegetativa aérea. Las condiciones específicas de cada experimento fueron las siguientes:

Experimento 1: las semillas de cada especie fueron sometidas a 45 tratamientos con una combinación factorial de cinco niveles de hidratación (0-24-48-72-96 h), tres de temperatura constante $\left(10-25-30{ }^{\circ} \mathrm{C}\right)$ y tres de iluminación diaria (0-12-24 h).

Experimento 2: este experimento está constituido por 12 tratamientos y fue diseñado a partir de los resultados obtenidos en el experimento 1. Para los seis primeros tratamientos se utilizó un período de hidratación constante de 96 h, con dos niveles 
de temperatura $\left(10\right.$ y $\left.25{ }^{\circ} \mathrm{C}\right)$, tres de iluminación (0-12-24 h). Los seis tratamientos restantes se mantuvieron expuestos a idénticas condiciones, con solo modificación en la temperatura entre la etapa de hidratación y de germinación de 10 a $25^{\circ} \mathrm{C}$ y de 25 a $10^{\circ} \mathrm{C}$.

Experimento 3: debido a la nula respuesta de la especie $S$. litoralis (ver resultados), se efectuó adicionalmente un tercer experimento solo con semillas de esta especie. Este experimento consistió en 30 tratamientos, donde se utilizó en cada uno de ellos 100 semillas de $S$. litoralis, divididas en cuatro réplicas de 25 semillas. Las semillas fueron hidratadas inicialmente por $96 \mathrm{~h}, \mathrm{y}$ posteriormente colocadas en cápsulas petri, donde se aplicaron los siguientes tratamientos: (a) hidratación durante todo el experimento (hidratación de las semillas cada 48 h), (b) hidratación/deshidratación (hidratación de las semillas cada 96 h), (c) escarificación manual/ deshidratación (cada $48 \mathrm{~h}$ se escarificaban manualmente y se hidrataban cada 96 h), (d) escarificación manual/hidratación (cada 48 h se escarificaban manualmente y se hidrataban las semillas) y (e) escarificación con sustrato húmedo cada 48 h e hidratación. La hidratación cada 48 h asegura una humectación constante de las placas. Las semillas que fueron hidratadas cada $96 \mathrm{~h}$ sufrieron un período de deshidratación natural por evaporación. La escarificación manual se realizó por abrasión con tela de tejido fino, bajo agua corriente. En la escarificación con sustrato, las semillas se colocaron en frascos plásticos con suelo del sector de colecta, humedecido hasta superar el punto de capacidad de campo y cada $48 \mathrm{~h}$ fueron puestas en un agitador mecánico por 30 minutos. A cada tratamiento se le aplicaron dos niveles de temperatura $\left(10\right.$ y $\left.25{ }^{\circ} \mathrm{C}\right)$ y tres de iluminación (0-12-24 h). Adicionalmente, se monitoreó visualmente el efecto producido en la testa de semillas incluidas en los tratamientos con escarificación manual y con sustrato, donde se extrajeron y observaron en una lupa estereoscópica cada $48 \mathrm{~h}$.

\section{Condiciones de germinación en laboratorio versus ambiente natural}

Después de realizados los tres experimentos germinativos en todas las especies en estudio, se compararon las condiciones de máxima germinación en laboratorio con las características ambientales de germinación natural. Las características ambientales de cada sitio de estudio se establecieron por observación en terreno y datos climáticos de las zonas de colecta.

Se examinaron las condiciones climáticas en las épocas de germinación de las especies en estudio y la textura del sustrato de los sitios donde habitan en forma natural y fueron contrastadas con los resultados de las condiciones de máxima germinación obtenidos en laboratorio. Los datos ambientales fueron obtenidos de centros meteorológicos cercanos a los lugares de colecta de las semillas y las condiciones de textura del suelo fueron observadas en terreno.

\section{Análisis estadístico}

Las respuestas germinativas se analizaron para cada especie mediante análisis de varianza factorial, considerando la germinación como variable dependiente. Se aplicó transformación angular a los datos para cumplir con el supuesto de distribución normal. Las diferencias encontradas en el análisis factorial se evaluaron con la prueba de Tukey $(\mathrm{P}<0,05)$.

\section{RESULTADOS}

\section{Prueba de viabilidad}

En la prueba de viabilidad pregerminativa, cinco de las especies en estudio presentaron una viabilidad superior al $90 \%$, exceptuando $S$. litoralis con un $82 \%$. La viabilidad promedio postgerminativa en semillas no germinadas en los diferentes tratamientos de todas las especies fue de un $85 \%$, excepto en los tratamientos de deshidratación en $S$. litoralis donde la viabilidad de las semillas se redujo a un $5 \%$.

\section{Experimento 1}

Las pruebas germinativas determinaron que en todas las especies estudiadas, el efecto hidratación, temperatura, iluminación y sus interacciones son altamente significativos 
excepto el factor iluminación para $P$. coerulea y Schizanthus litoralis (Tabla 2). La germinación superó el $90 \%$ en aquellos tratamientos que presentaban condiciones externas óptimas que estimularon la germinación de las semillas. Al analizar en forma individual cada factor externo y las respuestas germinativas de las especies estudiadas (no se considera $S$. litoralis por su baja germinación que fue menor al $5 \%$ ) se observa que: (a) hidratación, la máxima germinación se logró al superar un umbral de 96 h de hidratación, exceptuando $P$. amoena que requiere sobre $48 \mathrm{~h}$ de hidratación (Fig. 2A), (b) temperatura, en general los niveles de temperatura necesarios para iniciar el proceso germinativo están entre 10 y $25{ }^{\circ} \mathrm{C}$, inhibiéndose a los $30{ }^{\circ} \mathrm{C}$ (Fig. 2B). Específicamente, $C$. salsoloides y $P$. amoena, requieren una temperatura de $25{ }^{\circ} \mathrm{C}$, en cambio, L. purpurea, $P$. coerulea y $T$. plumosum solo $10{ }^{\circ} \mathrm{C}$ e (c) iluminación, el efecto de la iluminación sobre la germinación fue especie dependiente. Se puede inferir la existencia de dos grupos con respecto a requerimientos lumínicos: especies dependientes (L. purpurea, $P$. amoena y $T$. plumosum) especies no dependientes ( $L$. purpurea y P. coerulea) (Fig. 2C).

\section{Experimento 2}

Solo en dos de las especies estudiadas el efecto temperatura y sus interacciones fueron significativas $(C$. salsoloides y $P$. amoena). La única especie que presenta una escasa germinación (menor al $5 \%$ ) fue S. litoralis (Fig. 2D).

\section{Experimento 3}

La máxima germinación de $S$. litoralis fue sobre un $75 \%$ solo posterior a los tratamientos de escarificación manual y con sustrato a $10{ }^{\circ} \mathrm{C}$ y en oscuridad (Fig. 3 y 4). Las lesiones producidas en la testa de las semillas de $S$. litoralis, presentaron diferencias: la escarificación manual, genera un rápido adelgazamiento y una desaparición homogénea de la testa en un par de días, en cambio, en la escarificación por sustrato las lesiones son localizadas y se manifiestan en aproximadamente el triple del tiempo (Fig. 5). Las semillas que recibieron tratamientos de hidratación presentaron escasas respuestas germinativas, corroborando lo encontrado en los experimentos 1 y 2 . Las semillas expuestas a períodos de deshidratación no lograron germinar y el análisis de viabilidad postgerminativo reveló una disminución al $5 \%$ de su viabilidad.

TABLA 2

Análisis de varianza (valores de F) de la respuesta germinativa en condiciones de laboratorio de seis especies herbáceas de la zona árida de Chile (experimento 1); grados de libertad residual = 135

Analysis of variance of germination response under laboratory conditions of six herbaceous species of arid zone of Chile; residual degrees of freedom $=135$

\begin{tabular}{|c|c|c|c|c|c|c|c|}
\hline Variables & $\begin{array}{l}\text { Grados } \\
\text { libertad }\end{array}$ & C. salsoloides & P. amoena & L. purpurea & P. coerulea & T.plumosum & S. litoralis \\
\hline Temperatura & 2 & $165,71 *$ & $230,09 *$ & $1020,58^{*}$ & $321,27 *$ & $3984,85^{*}$ & $11,59 *$ \\
\hline Iluminación & 2 & $28,21 *$ & $41,63 *$ & $139,87^{*}$ & $0,69 \mathrm{~ns}$ & $94,29 *$ & $1,61 \mathrm{~ns}$ \\
\hline Hidratación & 4 & $603,02 *$ & $235,87 *$ & $463,94 *$ & $179,64^{*}$ & $753,62 *$ & $34,60 *$ \\
\hline Temperatura-iluminación & 4 & $61,9 *$ & $117,51 *$ & $87,51 *$ & $74,06 *$ & $103,89 *$ & $6,19 *$ \\
\hline Temperatura-hidratación & 8 & $42,25^{*}$ & $76,07 *$ & $35,88^{*}$ & $10,58^{*}$ & $180,35^{*}$ & $8,68 *$ \\
\hline Iluminación-hidratación & 8 & $20,73 *$ & $42,58^{*}$ & $25,05^{*}$ & $23,27 *$ & $20,60 *$ & $4,30 *$ \\
\hline $\begin{array}{l}\text { Temperatura-ilumina- } \\
\text { ción-hidratación }\end{array}$ & 16 & $23,67 *$ & $14,53^{*}$ & $20,04 *$ & $12,84 *$ & $19,65 *$ & $6,59 *$ \\
\hline
\end{tabular}

$(*)=\mathrm{P}<0,001 ; \mathrm{ns}=\mathrm{P}>0,05$ 

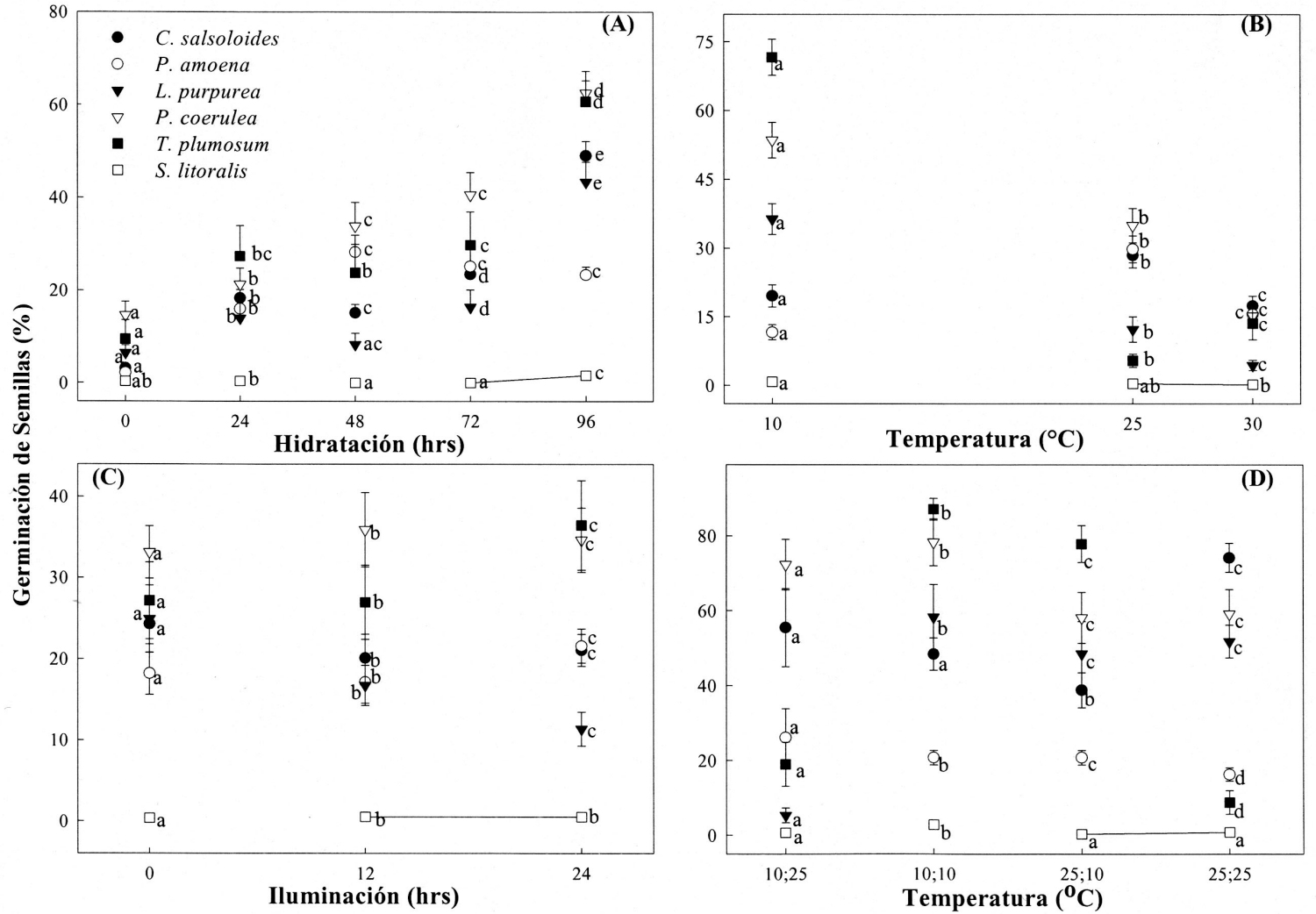

Fig. 2: Promedio germinativo en las especies en estudio expuestas a diferentes tratamientos germinativos con combinaciones factoriales de niveles de hidratación, temperatura e iluminación (experimentos 1 y 2).

Averages of germination of the species under study exposed to different germination treatments with factorial combinations of hydration, temperature and light levels (experiments 1 and 2).

Condiciones germinativas de laboratorio y naturales

Las combinaciones de factores externos donde se logró la mayor germinación en las seis especies en estudio, concuerdan con las condiciones ambientales donde las semillas logran germinar en forma natural (Tabla 3).

\section{DISCUSIÓN}

La zona norte de Chile, lugar de procedencia de las seis especies en estudio, presenta condiciones de aridez que impiden el crecimiento abundante de especies vegetales, exceptuando en temporadas donde se producen mayores precipitaciones. Los requerimientos de temperatura necesarios para la germinación de las especies vegetales herbáceas estudiadas están entre 10 y $25{ }^{\circ} \mathrm{C}$, decayendo bruscamente a los $30^{\circ} \mathrm{C}$, semejante a lo encontrado por Fausey \& Renner (1997) en la familia Poaceae. Las variaciones ocurridas en la capacidad germinativa de las semillas expuestas a tratamientos de bajas temperaturas $\left(10{ }^{\circ} \mathrm{C}\right)$, pueden explicarse por la desaparición de inhibidores y el incremento de la activación hormonal (Bewley \& Black 1978, Taylorson \& Hendricks 1977, Dewlin 1982, Rodríguez et al. 1998), o bien por ser especies asociadas a germinaciones de invierno, donde las bajas temperaturas rompen el período de latencia (Milberg \& Anderson 1998). El factor iluminación, permitió subdividir a las seis especies vegetales en dos subgrupos dependientes o no dependientes de los requerimientos lumínicos. 


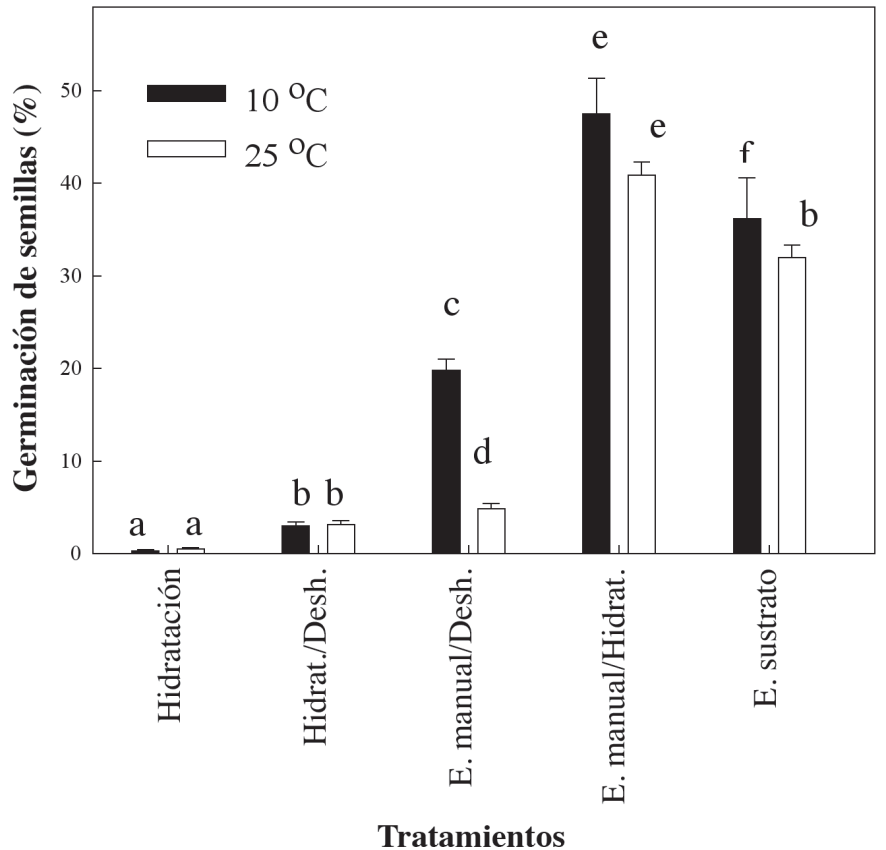

Fig. 3: Promedio germinativo en Schizanthus litoralis, obtenido con diferentes tratamientos expuestos a dos niveles de temperatura $\left(10\right.$ y $\left.25^{\circ} \mathrm{C}\right)$ (experimento 3$)$.

Average of germination percentage in Schizanthus litoralis, obtained with different treatments of two temperature levels $\left(10\right.$ and $\left.25^{\circ} \mathrm{C}\right)$ (experiment 3$)$.

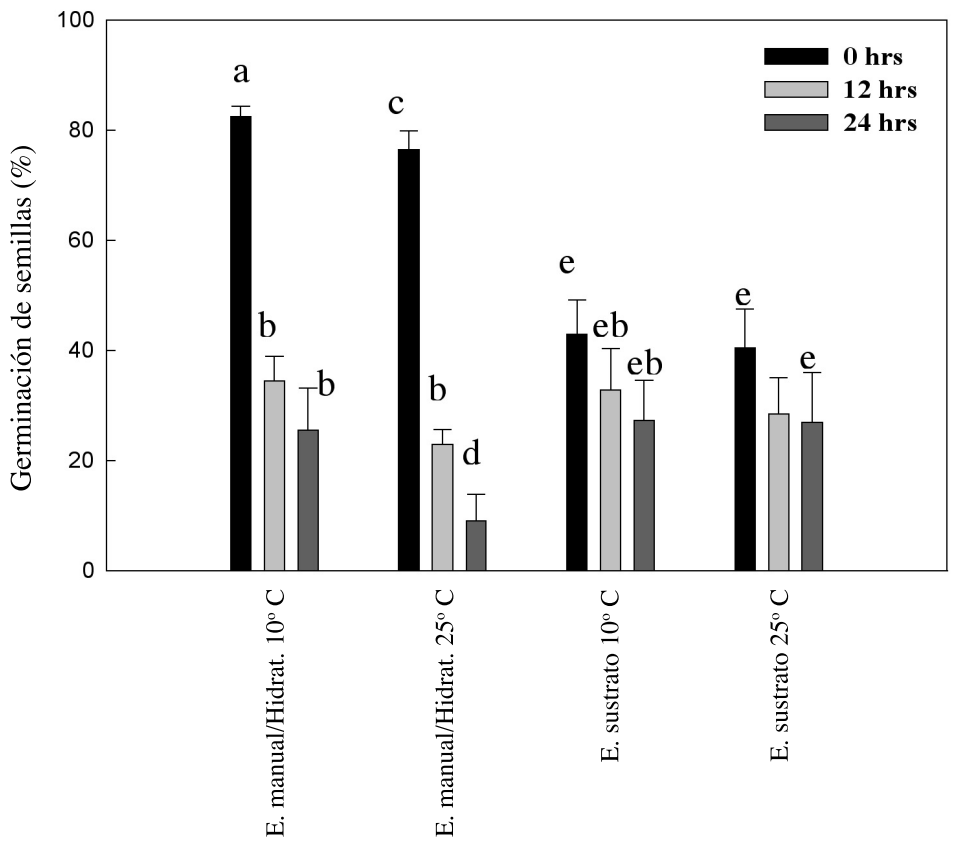

Tratamientos

Fig. 4: Promedio germinativo en Schizanthus litoralis, obtenido con diferentes tratamientos expuestos a dos niveles de temperatura $\left(10\right.$ y $\left.25^{\circ} \mathrm{C}\right)$ y combinaciones de luminosidad (experimento 3 ).

Average of germination percentage in Schizanthus litoralis, obtained with different treatments of two temperature levels $\left(10\right.$ and $\left.25^{\circ} \mathrm{C}\right)$ and combinations of light levels (experiment 3$)$. 

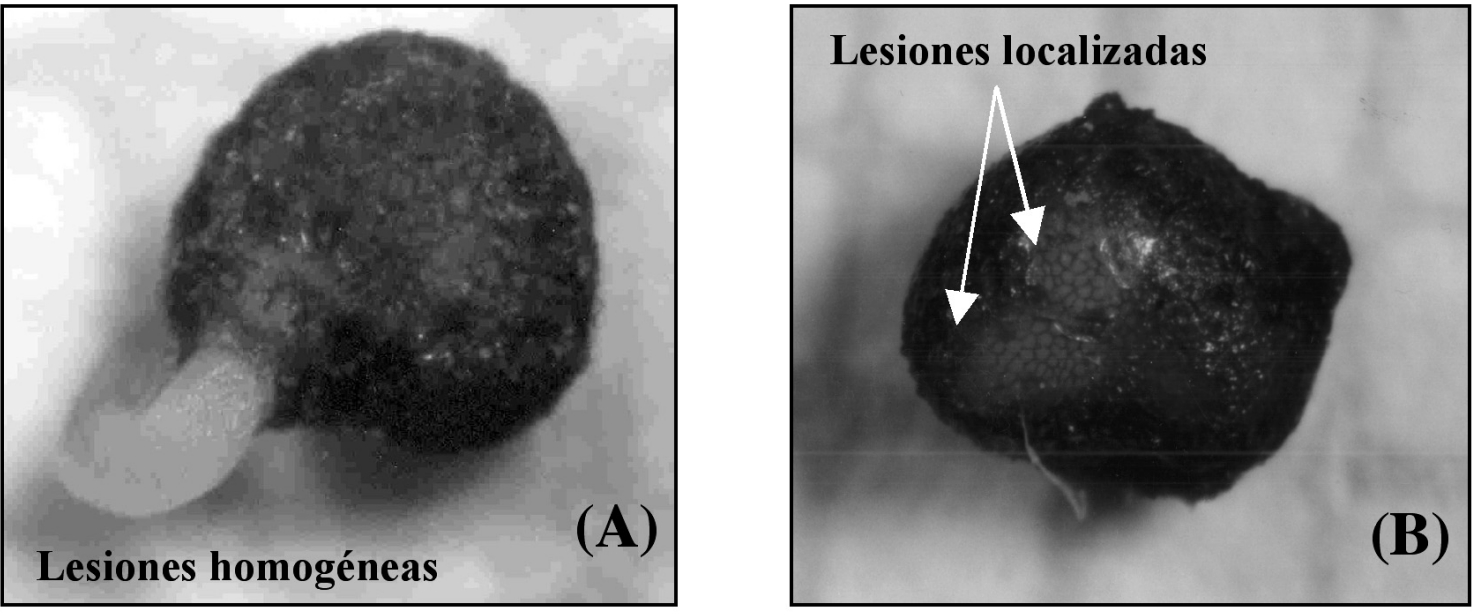

Fig. 5: Lesiones producidas en la testa de las semillas de S. litoralis, expuestas a diferentes mecanismos de escarificación, (A) manual (lesiones homogéneas en toda la testa en un par de días) y (B) sustrato (lesiones localizadas en el triple del tiempo) (experimento 3) (1 $\mathrm{mm}=---$ ).

Lesions produced on testa of $S$. litoralis seeds exposed to distinct scarification mechanisms, (A) manual (homogenous lesions over all testa within a couple of days) and (B) substrate (lesions located within tree-fold time) (experiment 3$)(1 \mathrm{~mm}=-$ -

TABLA 3

Comparación de condiciones ambientales de germinación natural y de laboratorio en seis especies de la zona árida de Chile (*previa escarificación)

Comparison of environmental conditions of both natural and laboratory germination of six species of arid zone of Chile (*previous scarification)

\begin{tabular}{|c|c|c|c|c|c|c|c|}
\hline \multirow[b]{2}{*}{ Especie } & \multicolumn{4}{|c|}{ Ambiental (natural) } & \multicolumn{3}{|c|}{ Laboratorio (>90\% germinación) } \\
\hline & Textura suelo & $\begin{array}{l}\text { Humedad e } \\
\text { iluminación }\end{array}$ & Período & $\begin{array}{l}\text { Rango tem- } \\
\text { peratura }\left({ }^{\circ} \mathrm{C}\right)\end{array}$ & $\begin{array}{c}\text { Hidratación } \\
\text { (h) }\end{array}$ & $\begin{array}{c}\text { Iluminación } \\
(\mathrm{h})\end{array}$ & $\begin{array}{c}\text { Temperatura } \\
\left({ }^{\circ} \mathrm{C}\right)\end{array}$ \\
\hline C.s. & Arenoso & Seco/soleado & Dic/ene & $10-30$ & 96 & 0 & 25 \\
\hline P. a. & Arenoso/arcilloso & Húmedo/sombrío & Jun/jul & $10-25$ & $48-96$ & 24 & 25 \\
\hline L. $p$. & Arenoso & Seco/soleado & Jun/jul & $7-16$ & 96 & 0 & 10 \\
\hline P.c. & Arenoso/arcilloso & Seco/soleado & Jun/jul & $7-16$ & 96 & 12 & 10 \\
\hline T. $p$. & Arenoso/arcilloso & Seco/soleado & Jun/jul & $7-16$ & 96 & 24 & 10 \\
\hline S. $l$. & Arenoso/arcilloso & Seco/soleado & Jun/jul & $>7<16$ & $96^{*}$ & $0 *$ & $10^{*}$ \\
\hline
\end{tabular}

Al recordar las interrogantes establecidas sobre si las respuestas germinativas eran comunes o específicas para las especies en estudio. Se logró inferir que el umbral de hidratación es similar en las especies estudiadas. Pero las combinaciones de los factores de temperatura e iluminación, donde se produce la máxima germinación es especie dependiente. La respuesta a la segunda interrogante sobre si las condiciones naturales de germinación concuerdan con las encontradas en laboratorio, se determinó alta congruencia. Las especies estudiadas habitan principalmente suelos arenosos, muy secos de alta evaporación, donde el agua escurre rápidamente (Agnew 1977, Rozzi et al. 1989, Kramer \& Boyer 1995, Marquet et al. 1998), por esta razón los umbrales de hidratación son 
altos. En los ambientes xéricos el inicio de los procesos germinativos, se relaciona más con eventos independientes, que con una sumatoria de pulsos variables e impredecibles de precipitación (Noy-Meir 1973, Schaffer \& Gadgil 1975), esto se debe a que las semillas de especies vegetales de zonas xéricas no germinan hasta que las condiciones ambientales sean las necesarias para asegurar el completo desarrollo de su ciclo biológico. Placea amoena presentó el menor umbral de hidratación de germinación, lo cual se relaciona con su hábitat, que es preferentemente suelo arcilloso, donde el riesgo de desecación es menor que en los suelos arenosos. A nivel de temperatura también existen concordancias con las condiciones reinantes en zonas xéricas estudiadas, donde las temperaturas fluctúan entre $\operatorname{los} 7$ y $25{ }^{\circ} \mathrm{C}$ en forma natural, lo que concuerda perfectamente con la mayor germinación en los niveles de temperatura entre 10 y $25{ }^{\circ} \mathrm{C}$, en el laboratorio, lo cual se relaciona, a su vez, con los niveles de humedad del suelo. La luminosidad requerida por las semillas es específica para cada especie.

Por lo tanto, la germinación artificial no surge de la elección de factores ambientales al azar, sino de una visión precisa y general de las condiciones del medio natural de germinación, que permiten clarificar y determinar en forma certera los tratamientos germinativos a escoger.

La inactividad de las semillas de $S$. litoralis, frente a los diversos tratamientos germinativos se debe a que sus semillas requieren de escarificación para germinar. La testa es una barrera física que impide la entrada de agua y por ello la germinación. Al comienzo de la germinación la testa debe sufrir alteraciones, como la disolución de los compuestos hidrosolubles (Bewley \& Black 1978) que genera pequeños sectores descubiertos por donde penetra el agua y se produce el intercambio gaseoso. Los experimentos realizados en $S$. litoralis, demostraron que mediante abrasión manual la semilla germina perfectamente. Al meditar sobre el proceso artificial de escarificación, surge la interrogante sobre qué mecanismo utilizan las semillas para hacerlo en forma natural. En este caso, la prueba de escarificación con sustrato del lugar de colecta, sugiere el efecto producido por los fuertes flujos de agua provenientes de precipitaciones, que arrastran las semillas y las golpean contra los granos del sustrato, causando el deterioro de la testa y dejando zonas expuestas y aptas para la captación de agua e intercambio gaseoso. Tales respuestas se han comprobado en estudios con otras especies vegetales realizados por Went (1953) y Gutterman $(1994,1996)$, donde la testa es dañada por el golpe de las semillas con el sustrato.

Los escasos resultados germinativos obtenidos en los tratamientos de deshidratación, se relacionan con que los procesos de hidratación-deshidratación-rehidratación son muy riesgosos para las semillas en general. Al hidratarse una semilla, se inicia un aumento en las reacciones enzimáticas que estimulan la germinación, pero al deshidratarse bruscamente los procesos germinativos se detienen y se provocan daños en la testa y a nivel embrional, disminuyendo considerablemente la viabilidad de la semilla (Bewley \& Black 1982).

\section{AGRADECIMIENTOS}

Paola Jara es Becaria Doctoral de la Comisión Nacional de Ciencia y Tecnología y Beca AT 24060111. Agradecemos al Departamento de Biología y al Herbario de la Universidad de La Serena. También se agradece al Proyecto Milenio P02-051-F (ICM) por apoyo en la etapa final de redacción del manuscrito.

\section{LITERATURA CITADA}

ALPERS C \& G BRIMHALL (1988) Middle Miocene climate change in the Atacama Desert, Northern Chile: Evidence from supergene mineralization at La Escondida. The Geological Society of America Bulletin 100: 1640-1656.

AGNEW ADQ (1997) Switches, pulses and grazing in arid vegetation. Journal of Arid Environments 37: 609617.

ARROYO MTK, JJ ARMESTO \& C VILLAGRÁN (1981) Plant phenological patterns in the high Andean Cordillera of central Chile. Journal of Ecology 61: 205-233.

ARROYO MTK, FA SQUEO, JJ ARMESTO \& C VILLAGRAN (1988) Effects of aridity on plant diversity in the northern Chilean Andes: Results of a natural experiment. Annals of the Missouri Botanical Garden 75: 55-78.

BAILI L \& M FOLEY (1997) Genetic and molecular control of seed dormancy. Elsevier Science 2:384389.

BASKIN JM \& CC BASKIN (1978) Seasonal changes in the germination response of Cyperus inflexus seeds to temperature and their ecological significance. Botanical Gazette 139:231-235. 
BASKIN JM \& CC BASKIN (1981) Seasonal changes in the germination responses of buried Lamium amplexicaule seeds. Weed Research 21: 299-306.

BASKIN JM \& CC BASKIN (1984) Effects of temperature during burial on dormant and non-dormant seeds of Lamium amplexicaule L. and ecological implications. Weed Research 24: 333-339.

BASKIN JM \& CC BASKIN (1993) Annual seed dormancy cycle in two desert winter annuals. Journal of Ecology 81: 551-556.

BASKIN JM \& CC BASKIN (1998) Seeds: ecology, evolution, and biogeography, dormancy and germination. Academic Press, San Diego, California, USA. 666 pp.

BETANCOURT JL, C LATORRE, JA RECH, J QUADE \& KA RYLANDER (2000) A 22,000-year record of monsoonal precipitation from northern Chile's Atacama desert. Science 289: 1542-1546.

BEWLEY J \& M BLACK (1978) Physiology and biochemistry of seeds. Volume I. Springer-Verlang, Berlin, Germany. 363 pp.

BEWLEY J \& M BLACK (1982) Physiology and biochemistry of seeds. Volume II. Sprnger-Verlag, Berlin, Germany. 315 pp.

BOOTH R \& G HENDY (1993) Seed viability and germination. En: Hendry GAF \& JP Grime (eds) Methods in comparative plant ecology: a laboratory manual: 10-13. Chapman \& Hall, London, United Kingdom.

BORGEL R (1973) The coastal desert of Chile. En: Amiran DHK \& AW Wilson (eds) Coastal desert: their natural and human environments: 111-114. University of Arizona Press, Tucson, Arizona, USA.

CAVIEDES C (1973) A climatic profile of the north Chilean desert at latitude $20^{\circ} \mathrm{S}$. En: Amiran DHK \& AW Wilson (eds) Coastal desert: their natural and human environments: 115-121. University of Arizona Press, Tucson, Arizona, USA.

CEPEDA J (1995) Síntesis ecológica del desierto costero peruano-chileno. Revista Interna de Investigación y Desarrollo, Universidad de La Serena (Chile) 1: 414.

DEWLIN R (1982) Fisiología vegetal. Omega, Barcelona, España. 517 pp.

EVENARI M \& Y GUTTERMAN (1976) Observations on the secondary succession of three plant communities in the Negev Desert, Israel. Artemisietum herbae-albae. En: Gutterman Y (1994) Strategies of seed dispersal and germination in plants inhabiting desert. The Botanical Review 60:371-425.

FAUSEY JC \& KA RENNER (1997) Germination, emergence, and growth of giant foxtail (Setaria faberi) and fall panicum (Panicum dichotomiflorum). Weed Science 45: 423-425.

FENNER M (1985) Seed ecology. Chapman and Hall, London, United Kingdom. 151 pp.

FROUD-WILLIAMS R, D DRENNAN \& $R$ CHANCELLOR (1984) The influence of burial and dry-storage upon cyclic changes in dormancy, germination and response to light in seeds of various arable weeds. New Phytologist 96: 473-481.

GENGLER-NOWAK KM (2002) Reconstruction of the biogeographical history of Malesherbiaceae. Botanical Review 68: 171-188.

GREGORY-WODZICKI KM (2000) Uplift history of the central and northern Andes: a review. The Geological Society of America Bulletin 112: 10911105 .
GUTTERMAN Y (1994) Strategies os seed dispersal and germination in plants inhabiting desert. The Botanical Review 60: 371-425.

GUTTERMAN Y (1996) Temperature during storage, light and weting affecting caryopses germinability of Schimus arabicus, a common desert annual grass. Journal of Arid Environments 33: 73-85.

HERBERT G \& HG Baker (1972) Seed weight in relation to environmental conditions in California. Ecology 53: 997-1010

INOUYE R (1991) Population biology of desert annual plants. En: Polis GA (ed) The ecology of desert communities: 27-54. University of Arizona, Tucson, USA.

JONES HG (1992) Plants and microclime. Cambridge University Press, Cambridge, United Kingdom. 236 pp.

KARSSEN C (1980) Enviromental conditions and endogenous mechanisms involved in secondary dormancy of seds. Israel Journal Botanical 19: 4564.

KEMP P (1989) Seed banks and vegetation processes in deserts. En: Leck MA, VT Parker \& RL Simpson (eds) Ecology of soil seed bank: 257-281. Academic Press, San Diego, California, USA

KEYA G (1997) Environmental triggers of germination and phenological events in an arid savannah region of northern Kenya. Journal of Arid Environments 37: 91-106.

KOCHMER JP \& JS ANDEL (1986) Water relations of plants and soils. Academic Press, San Diego, California, USA. $495 \mathrm{pp}$

KRAMER P \& J BOYER (1995) Water relation of plants and soil. Academic Press, Orlando, Florida, USA. $495 \mathrm{pp}$

LORIA M \& I NOY-MEIR (1979) Dynamics of some annual populations in a desert loess plain. Israel Journal Botanical 28: 211-225.

LYDOLPH PE (1973) On the causes of aridity along a selected group of coasts. En: Amiran DHK \& AW Wilson (eds) Coastal desert: their natural and human environments: 67-72. University of Arizona Press, Tucson, Arizona, USA.

MARQUET PA, F BOZINOVIC, GA BRADSHAW, C CORNELIUS, H GONZÁLEZ, JR GUTIÉRREZ, ER HAJEK, JA LAGOS, F LÓPEZ-CORTÉS, L NÚNEEZ, EF ROSELLO, C SANTORO, H SAMANIEGO, VG STANDEN, JC TORRESMURA \& FM JAKSIC (1998) Los ecosistemas del desierto de Atacama y área andina adyacente en el norte de Chile. Revista Chilena de Historia Natural 71: 593-617.

MARTICORENA C \& M QUEZADA (1985) Catálogo de la flora de Chile. Gayana Botánica (Chile) 42: 1155.

MAYER A \& A POLJAKOFF (1989) The germination of seed. Pergamon Press, Oxford, United Kingdom. $270 \mathrm{pp}$

MILBERG P \& L ANDERSSON (1998) Does cold stratification level out differences in seed germinability between populations? Plant Ecology 134: 225-234

MOORE R (1973) Tetrazolium staining for assessing seed quality. En: Heydecker W (ed) Seed ecology: 347366. Butterworths, London, United Kingdom.

NOY-MEIR I (1973) Desert ecosystems: environment and producers. Annual Review of Ecology and Systematics 4: 25-51

PALLAS JE, JR STANSELL \& RR BRUCE (1997) Peanut seed germination as related to soil water regime 
during pod development. Agronomy Journal 69: 381-383.

RODRÍGUEZ A, E JACOBO \& V DEREGIBUS (1998) Germination behavior of Italian ryegrass in flooding pampa rangelands. Seed Science Research 8: $521-528$

ROMERO H (1985) Geografía de los climas. Colección Geografía de Chile. Instituto Geográfico Militar, Santiago de Chile, Chiel. 243 pp.

ROZZI R, R MOLINA \& P MIRANDA (1989) Microclima y periodos de floración en laderas de exposición ecuatorial y polar en los Andes de Chile central. Revista Chilena de Historia Natural 62: 75-84.

SCHAFFER W \& M GADGIL (1975) Selection for optimal life histories in plants. En: Cody M \& JM Diamond (eds) Ecology and evolution of communities: 142-157. Belknap Press of Harvard University Press, Cambridge, Massachusetts.

SQUEO FA, JR EHLERINGER, NC OLIVARES \& G ARANCIO (1994) Variation in leaf level energy balance components of Encelia canescens

along a precipitation gradient in north-central Chile. Revista Chilena de Historia Natural 67: 143-155.

Editor Asociado: Javier Figueroa

Recibido el 11 de agosto de 2005; aceptado el 3 de marzo de 2006
SQUEO FA, F RADA, CE GARCÍA, ME PONCE, AL ROJAS \& A AZÓCAR (1996) Cold resistance mechanisms in high desert Andean plants. Oecologia 105: 552-555.

SQUEO FA, G ARANCIO \& J GUTIÉRREZ (2001) Libro de la flora nativa y de los sitios prioritarios para su conservación: Región de Coquimbo. Ediciones de la Universidad de La Serena, La Serena, Chile. 372 $\mathrm{pp}$

TAYLORSON R \& B HENDRICKS (1977) Dormancy in seeds. Annual Review of Plant Physiology 28: 331354.

TOLEDO X \& E ZAPATER (1989) Geografía general y regional de Chile. Editorial Universitaria, Santiago, Chile. 443 pp.

VEGIS A (1964) Dormancy in higher plants. Annual Review of Plant Physiology 15: 185-215.

WENT F (1953) The effects of rain and temperature on plant distribution in the desert. En: Gutterman Y (1994) Strategies of seed dispersal and germination in plants inhabiting deserts. The Botanical Review 60: $371-425$ 
